\title{
Analyst
}

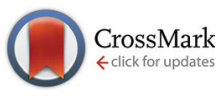

Cite this: Analyst, 2015, 140, 6217

Received 21st April 2015

Accepted 24th July 2015

DOI: 10.1039/c5an00772k

www.rsc.org/analyst

\section{Analysis of mass-limited mixtures using supercritical-fluid chromatography and microcoil NMR}

\author{
Michael C. D. Tayler, ${ }^{\star}$ S. (Bas) G. J. van Meerten, Arno P. M. Kentgens and \\ P. Jan M. van Bentum*
}

A protocol is presented for offline microfluidic NMR analysis hyphenated with supercritical chromatographic separation. The method demonstrates quantitative detection with good sensitivity. Typical sample amounts of 10 nanomoles can be detected in a fast and cost-effective manner.

For several decades, liquid-phase chromatography (LC) coupled with nuclear magnetic resonance (NMR) spectroscopy has formed a powerful tool for analysing mixtures. Physical separation of molecular components is followed by spectral examination of their chemical composition. ${ }^{1-3} \mathrm{NMR}$ is unbiased, quantitative, and requires no external calibration or standards, making it the preferred technique for resolving molecular structure and isomerism, especially in cases where little or no prior knowledge of the system exists. Yet an NMR experiment is often slow, insensitive and most of the present commercial apparatus incurs high setup and operational costs. These features make LC-NMR rarely a first-choice analysis option in medium- to high-throughput settings when compared with other techniques, for instance LC-mass spectrometry (LC-MS).

This work aims to demonstrate that with help from costand time-saving innovations, chromatography-NMR hyphenation may become feasible for routine analysis of mass quantities of the order 10 nanomole $(<1 \mu \mathrm{g})$. For low-mass samples, the most efficient NMR sensor is a microcoil. ${ }^{4,5}$ It is beneficial to concentrate the sample into the smallest possible volume and maximise the reciprocity principle that applies to inductive detection. Small sample volumes facilitate the choice of using compact, less homogeneous magnets and also smaller quantities of deuterated solvents are needed. One of the main challenges facing microfluidic NMR detection using microcoils is minimising line broadening due to magnetic susceptibility distortions. ${ }^{6,7}$ The innovation of flat, linear radiofrequency stripline structures ${ }^{8,9}$ is a promising design for reducing these dis-

Institute for Molecules and Materials, Radboud University, Nijmegen 6525AJ, Netherlands.E-mail:mcdt2@cam.ac.uk, j.vanbentum@science.ru.nl; Tel: +31243652387 tortions by (i) using a simpler topology (ii) orienting the inductor's axis parallel to the static field generated by the NMR magnet, (iii) being easily scalable to sample dimensions. The striplines make high-resolution spectroscopy possible on volumes of order $100 \mathrm{~nL}$ to a few $\mu \mathrm{L}$, with sensitivities of 0.1 nanomole ${ }^{1} \mathrm{H} \sqrt{\mathrm{Hz}} .{ }^{10}$ In previous work, this utility has been demonstrated in the of profiling mouse cerebro-spinal fluids ${ }^{11}$ and in hyphenation with capillary electrophoresis-solid phase extraction (CE-SPE-NMR). ${ }^{12}$

Supercritical fluid chromatography (SFC) is chosen as a time- and cost-efficient companion to stripline NMR. Lowviscosity solvent mixtures based on supercritical-phase carbon dioxide $\left(\mathrm{sc}-\mathrm{CO}_{2}, P>70 \mathrm{bar}, T>40{ }^{\circ} \mathrm{C}\right.$ ) permit flow rates about 10 times higher than those used in normal phase $\mathrm{LC}^{13,14}$ due to the gas-like mobility of $\mathrm{sc}-\mathrm{CO}_{2}$ at liquid-like densities of the medium. The sc- $\mathrm{CO}_{2}$ solvent basis greatly reduces quantities of organic solvent needed, fulfilling current "green" policy objectives. It may also avoid derivatization steps sometimes necessary in LC and offers a natural integration with supercritical-fluid extraction (SFE). ${ }^{15}$ As these features are becoming increasingly popular, separation technology using $\mathrm{sc}-\mathrm{CO}_{2}$ is emerging from its "niche" and rivalling traditional LC and gas-phase chromatography. ${ }^{16,17}$

As a proof-of-principle demonstration of analytical scale SFC-NMR we separated and identified the components of a mixture of vitamin $\mathrm{E}$ (tocopherols). Isomers of vitamin $\mathrm{E}$ arise due to various aryl methylation patterns as illustrated in Fig. 1(a).

We prepared a vitamin E test mixture by diluting a commercial sample (Sigma Aldrich, CAS 1406-66-2, specified as not less than $80 \% \beta, \gamma$ and $\delta$ isomers) at $2 \mathrm{mg} \mathrm{mL}^{-1}$ in toluene. A $3 \mu \mathrm{L}$ aliquot of this solution - corresponding to $6 \mu \mathrm{g}$ of the original mixture and an upper bound of 14 nanomole tocopherols - was injected into a Waters Acquity ${ }^{\mathrm{TM}} \mathrm{UPC}^{2}$ instrument (Waters, Milford, CA). ${ }^{17}$ A $100 \mathrm{~mm} \times 1.7 \mu \mathrm{m}$ sphericalpacked BEH (bridged ethyl hybrid) chromatography column was used, running at a constant 120 bar backpressure and a solvent $2: 98 \mathrm{v} / \mathrm{v} \mathrm{MeOH}: \mathrm{CO}_{2}$ (0 minutes) to $5: 95 \mathrm{MeOH}: \mathrm{CO}_{2}$ (4 minutes) flowing at $2000 \mu \mathrm{L} \mathrm{min}^{-1} .{ }^{16}$ The chromatograph in Fig. 1(b) displays absorbance at $222 \mathrm{~nm}$ from the instrument's 
<smiles>[R]c1c([R])c2c(c([R])c1O)CC[C@@](C)(CCC[C@H](C)CCC[C@H](C)CCC(C)C)O2</smiles>

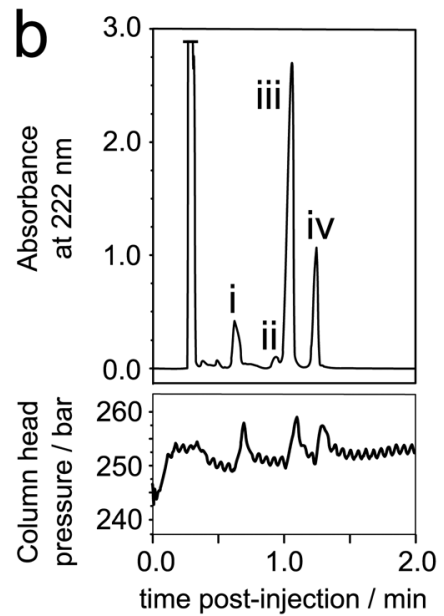

Fig. 1 Separation of the four tocopherol isomers (vitamin E) using SFC: (a) molecular structures of the tocopherols; (b) $222 \mathrm{~nm}$ absorbance and column head pressure versus retention time following the method described in the main text. Minor disturbances in the chromatography column head pressure occur following ejection of the $\alpha, \gamma$ and $\delta$ fractions. The absorbance at 0.33 minutes results from the toluene in which the mixture was dissolved before injection.

post-column photodiode array detector (PDA), showing four peaks baseline-resolved from one another within 1.5 minutes from sample injection. This is significantly faster than normal LC, where complete separation of the fractions requires some tens of minutes. ${ }^{18}$

Fig. 2 shows how the UPC ${ }^{2}$ instrument was modified for collection of the separated fractions. Part (a) illustrates regular
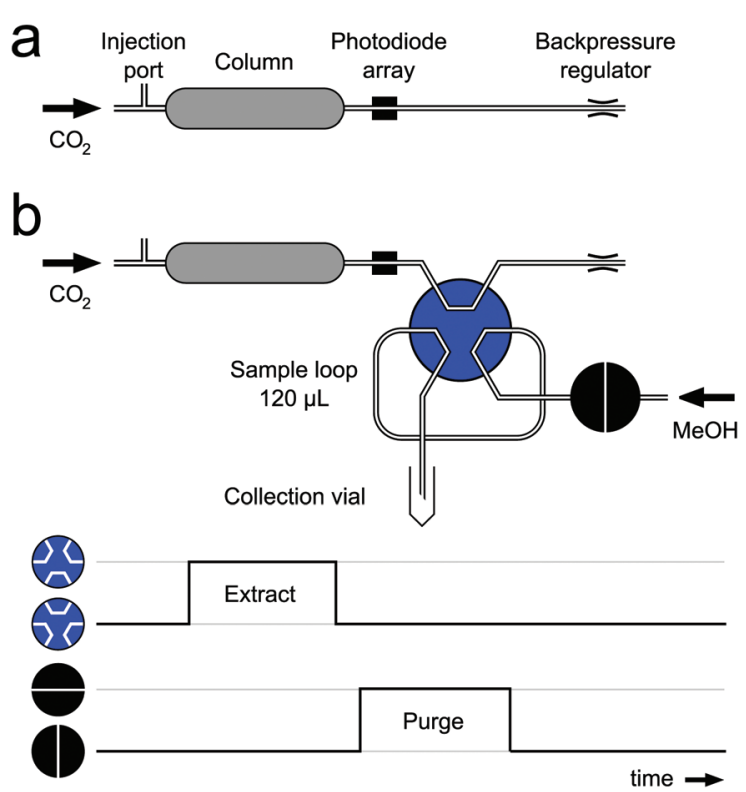

Fig. 2 Schematic view of the Waters Acquity ${ }^{\mathrm{TM}} \mathrm{UPC}^{2}$ instrument (a) from the manufacturer and (b) modified for fraction collection. The graph indicates the valve positions during one ejection cycle. operation of the machine. Two high-pressure binary-position valves (Vici Valco Inc.) were added, to be switched as shown in part (b). When a chromatographic fraction of interest is detected at the PDA, the 6-port (blue) valve is rotated by 60 degrees, to divert the flow into a side loop. When the loop is full, or when the fraction of interest has finished passing the PDA detector, the valve is returned to its original position. On returning the valve the side storage loop opens to atmospheric pressure and its high-pressure content is ejected into a disposable $500 \mu \mathrm{L}$ Eppendorf vial. The $\mathrm{CO}_{2}$ rapidly evaporates, depositing the concentrated extract. While the ejection is occuring, the second (black) valve is switched so that a small volume of methanol (LC-grade) runs through the loop. This (i) ensures all material is flushed into the collection vial so that the sample loop is clean for the next ejection and (ii) minimises disturbance to the chromatography column pressure during the next cycle, as the loop re-fills with an incompressible fluid. Control of the valves is managed by an Arduino UNO microcontroller ${ }^{19}$ interfaced with the I/O channels of the PDA and injector units. A short $\mathrm{C}++$-based program was written to trigger the ejections in various circumstances: (i) a given delay after sample injection; (ii) on exceeding a user-defined PDA absorbance threshold at wavelengths $300-800 \mathrm{~nm}$; (iii) on exceeding a user-specified time derivative in PDA absorbance. Manual sample collection yields using this setup are $>90 \%$, which we determine by comparing the UV spectra (Varian Cary 400) of a single-component solution before and after separation-ejection.

Tocopherol fractions were collected by triggering the ejection valve system at 0.2 PDA absorbance units. The sample loop was a 1.2 metre length of 0.062 inch o.d./0.015 inch i.d. PEEK tubing (internal volume $120 \mu \mathrm{L}$ ). At the flow rate $1500 \mu \mathrm{L}$ $\mathrm{s}^{-1} \mathrm{CO}_{2}$ this corresponds to a filling time of 6 seconds, enough to capture the entire chromatographic peak. A $250 \mathrm{~ms}$ delay between loop depressurisation and purging avoids any possibility of flow into the purge solvent reservoir. The line was purged at a flow rate of $5000 \mu \mathrm{L} \mathrm{min}{ }^{-1}$, resulting in $\approx 50 \mu \mathrm{L}$ additional methanol in the collection vial with each ejection.

The finite duration of each ejection-purge cycle imposes a dead-time ( $\approx 3$ seconds) between which fractions may be collected. Due to this limitation it was not possible to collect fractions (ii) and (iii) within the same "run". A more advanced setup may in future allow fractions to be collected with zero dead time, e.g. by operating two ejection loops in duplex. In the present example, only fractions from peaks (i), (iii) and (iv) could be collected within a single run. The isomer giving peak (ii) was collected during a second run using a timed trigger at 55 seconds. Note that this fraction is very close to the detection limit in the chromatogram. As an example of the possibility to screen also for low concentration components, possibly without a suitable UV chromatographic response, we chose to collect a summation of 10 consecutive runs, timed at the same interval. The short SFC separation time and low solvent consumption favour this practice.

The fractions were prepared for NMR analysis by removing the excess methanol under vacuum-centrifugation (10 minutes), 
yielding oils. These were each dissolved in $0.5 \mu \mathrm{L} 99.9 \%$ $\mathrm{d}_{4}$-methanol. A bench microscope was used to observe the dissolving process. Immediately after dissolution, the solutions were inserted by capillary suction into a $10 \mathrm{~cm}$ section of $250 \mu \mathrm{m}$ i.d./360 $\mu \mathrm{m}$ o.d. fused-silica capillary tubing (PolyMicro Technologies). Approximately $1 \mu \mathrm{L}$ fluorocarbon (FC-40), equal to approximately $2 \mathrm{~cm}$ of the capillary tube length, was inserted between each methanol plug in order to match magnetic susceptibility as well as isolate the fractions from one another. The capillary tube was then sealed at each end with optical glue.

Fig. 3 displays pulse-acquire ${ }^{1} \mathrm{H}$-NMR spectra of the fractions recorded at $600 \mathrm{MHz}$ Larmor frequency (14.1 tesla) using a house-built stripline probe interfaced with a Varian VNMRS solids spectrometer operated without a field lock or temperature stabilization system. The probe follows a design similar to that described in our previous work, ${ }^{12}$ with the stripline geometry optimised for detection over an active volume of $150 \mathrm{~nL}$ (active strip length $=4 \mathrm{~mm}$, width $=1 \mathrm{~mm}$ ). The single-shot mass sensitivity was calibrated for the room-temperature probe in the region of $3 \times 10^{13}{ }^{1} \mathrm{H}$ spins per square-root $\mathrm{Hz}$ receiver bandwidth. With minor shim adjustments to the magnet, spectral resolution circa $2 \mathrm{~Hz}$ full-width halfmaximum (FWHM) was achieved. Spectra in the figure are each the sum of 500 NMR transients recorded with a 90 degree flip angle pulse and 2 seconds relaxation/solvent presaturation delay, taking approximately 25 minutes experiment time per sample.

The measured NMR spectra show features that allow clear differentiation of the vitamin $\mathrm{E}$ isomers and prove their clean isolation from the starting mixture.

The fractions can be readily assigned with the known tocopherol isomers based on the number of aryl-H and

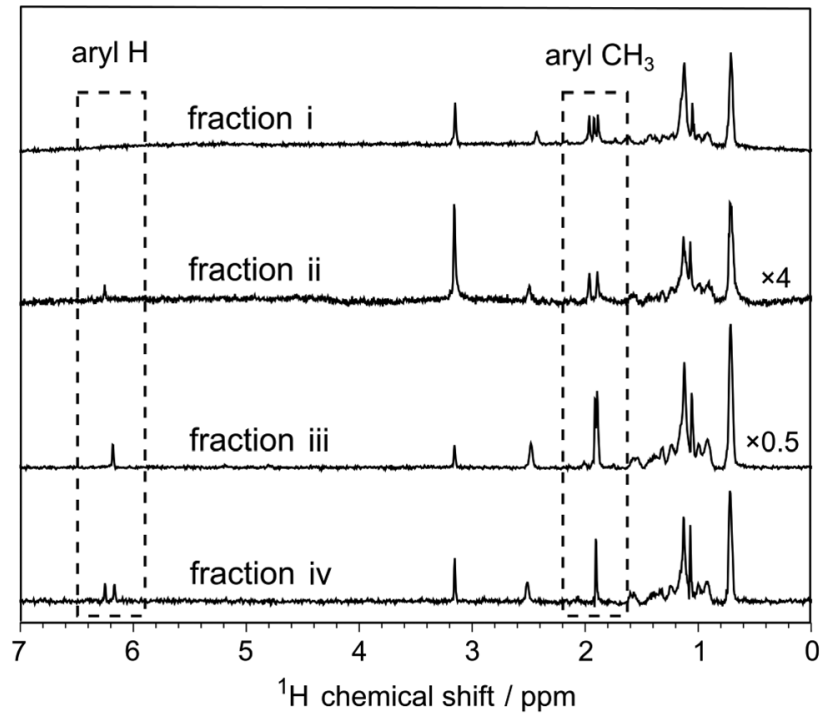

Fig. $3{ }^{1} \mathrm{H}$-NMR spectra of the ejected tocopherol fractions. Highlighted are the aryl substituents that differ between the isomers. aryl-methyl group resonances (dashed outline, Fig. 3). ${ }^{18,20}$ Fraction (i), with three methyl groups, is unambiguously $\alpha$-tocopherol, and fraction (iv) must contain $\delta$-tocopherol - it is noted that the apparent lack of splitting in the two aryl proton resonances is confirmation that these nuclei are not adjacently located on the ring. Fractions (ii) and (iii) each contain one of the aromatic hydrogens in (iv) and therefore must be $\beta$-tocopherol and $\gamma$-tocopherol. A definitive assignment can be made using the methyl resonance of $\delta$-tocopherol, which is common in all of the isomers; (ii) $=\beta$, (iii) $=\gamma$. Alternatively one may resolve the $\beta$ and $\gamma$ isomers by seeking an Overhauser cross-relaxation effect between the aryl and methyl protons.

To demonstrate spectral assignment in a system with greater molecular complexity, we separated a mixture containing the two steroid isomers cortisone and aldosterone (both $\mathrm{C}_{21} \mathrm{H}_{28} \mathrm{O}_{5}$, see Fig. 4(a)). This mixture was prepared by dissolving the steroids together, without derivatization, at $2 \mathrm{mM}$ in 4:1 methanol: isopropanol. A $2 \mu \mathrm{L}$ aliquot of the solution, corresponding to 10 nanomol $(0.36 \mu \mathrm{g})$ of each steroid, was injected into the Waters SFC instrument running a $100 \mathrm{~mm} \times$ $1.7 \mu \mathrm{m} \mathrm{BEH}$ column and 120 bar backpressure. The solvent was $8: 92 \mathrm{v} / \mathrm{v} \mathrm{MeOH}: \mathrm{CO}_{2}$ (0 minutes) to $15: 85 \mathrm{MeOH}: \mathrm{CO}_{2}$ (4 minutes), with a flow rate $1500 \mu \mathrm{L} \mathrm{min}^{-1}$. The chromatograph in Fig. 4(b) displays two peaks, one from each isomer, with retention times (i) 2 minutes 47 seconds and (ii) 3 minutes 12 seconds. These fractions were collected during the same run and methanol was removed using the same procedure as for the tocopherols, yielding small quantities of white powder at the bottom of each vial. These solids were each dissolved in $0.5 \mu \mathrm{L} 99.9 \% \mathrm{~d}_{4}$-methanol and inserted into capillaries for NMR analysis in the same manner described earlier.

NMR spectra of the steroids are shown in Fig. 4(c). From the resonances due to the protons in the vicinity of carbons C18 and C21 we can identify fraction (i) as cortisone $\left(\mathrm{C}^{2} \mathrm{H}_{3}\right.$ near $0.6 \mathrm{ppm}$ ) and (ii) as aldosterone (C18HO hydrate proton near $5.2 \mathrm{ppm}$, geminal $\mathrm{C} 21 \mathrm{H} / \mathrm{H}^{\prime}$ pair near $3.2 \mathrm{ppm}$ ) in agreement with the reference spectra of the molecules from prior work. $^{21}$

We compared the performance of the stripline probe against a high-end commercial NMR probe available in our lab. Cortisone was separated from the abovementioned mixture with aldosterone, evaporated to dryness, dissolved in $200 \mu \mathrm{L} \mathrm{d}_{4}$-methanol and placed in a $5 \mathrm{~mm}$ o.d. Shigemi tube. The ${ }^{1} \mathrm{H}$-NMR spectrum was recorded at $600 \mathrm{MHz}$ using a Bruker TCI $5 \mathrm{~mm}$ cryoprobe optimised for ${ }^{1} \mathrm{H}$ detection. We observed that the cryoprobe outperforms the stripline probe in terms of spectral resolution by at least a factor of 3 , with linewidths below $1.0 \mathrm{~Hz}$ FWHM easily obtained by automatic gradient shimming. Despite the disparity in resolution, the overall signal-to-noise was comparable between the two probes with the stripline performing marginally better than the cryoprobe. The stripline probe also obtained a much better suppression of the solvent background signal, due to the approximately 500 times smaller sample volume used. 


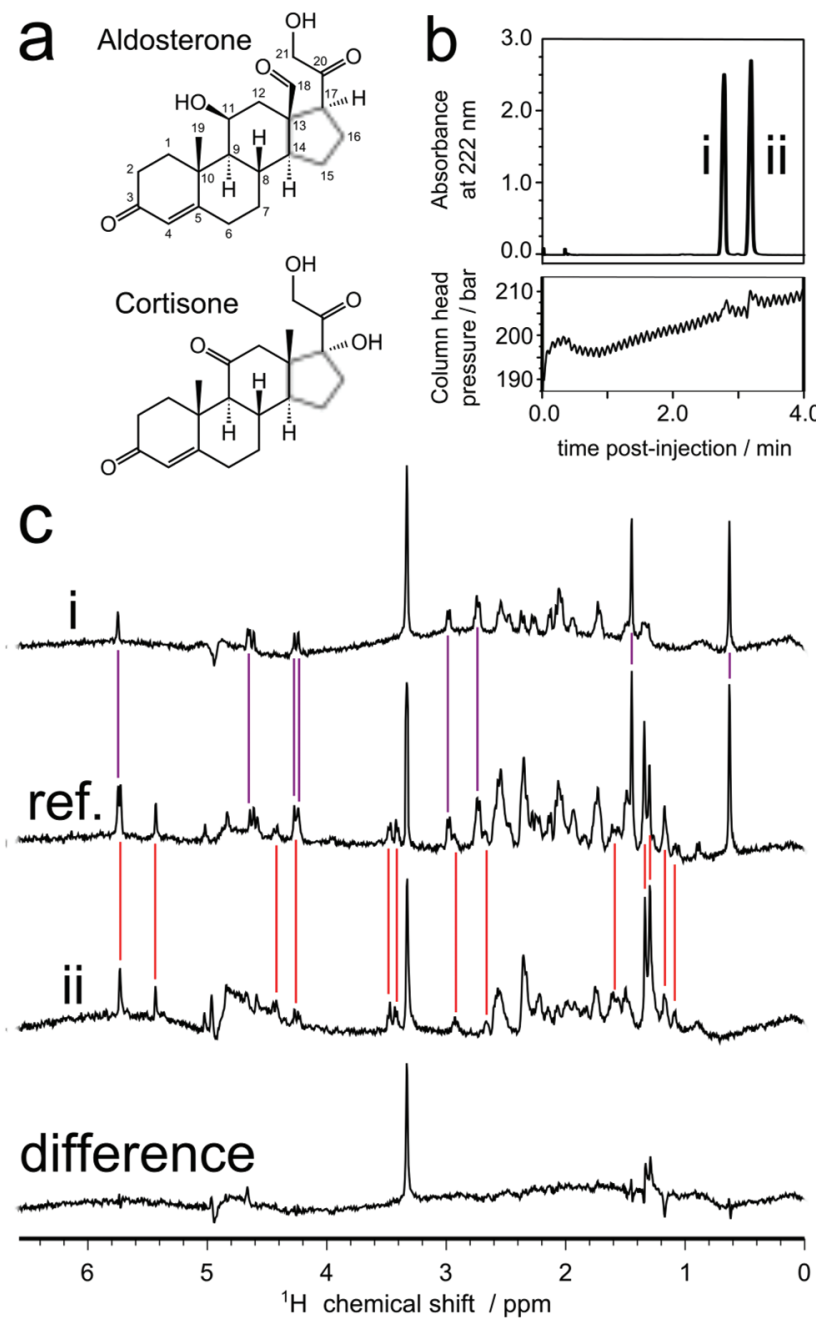

Fig. 4 SFC separation and NMR analysis of a cortisone-aldosterone mixture: (a) molecular structures of the steroids, which have the same molecular formula; (b) Chromatography data showing column pressure profile during collection of fractions (i) and (ii) from $2 \mu \mathrm{L}$ of the starting solution; (c) shows the HNMR spectrum of collected fractions (i) and (ii). The spectrum marked "ref." corresponds to the $2 \mu \mathrm{L}$ of the starting solution processed in the same way as fractions (i) and (ii), except omitting the chromatography step, for reference. The "difference" spectrum attempts to compare the relative substance quantities, as described in the main text.

Fig. 4(c) shows also a difference spectrum between spectra (i), (ii) and a reference spectrum of the unseparated mixture, "ref.", which is minimized by addition with weightings 1.27 , 1.20 and -1 , respectively. This shows that unknown components in the mixture must be below 1 nanomole. The subtraction procedure, however, indicates that the absolute intensity is not fully preserved, with only $80-90 \%$ of the original steroid components being recovered after the chromatographic separation and transferred to the active detection volume of the NMR probe. Further research is needed to verify whether a fully quantitative treatment is possible without additional calibration steps.
Improved quantitation of the substrates may be achieved by fully automating the SFC-NMR protocol so that it becomes "hands-free". In principle, instead of ejecting the chromatographic fractions into a vial, they may be collected, concentrated and stored on a solid-phase extraction column. They may then be dissolved in a proton-free solvent of choice and then fluidically shuttled to the NMR probe, providing a suitable method for automated sequential screening. A fully closed-system approach should eliminate sample loss and better suit mixtures containing volatile compounds. Using the present protocol of loading different sample fractions in a single capillary provides a compact means of storage, and makes samples readily available for future reference. As the NMR analysis is non-invasive, the analysis may be reproduced at any time. If a suitable sample translation system were to be included in the probe, all fractions could be measured in sequence under full computer control to provide a fast quantitative aquisition protocol, including multiple averaging or $2 \mathrm{D}$ analysis, without the usual $T_{1}$ delays. This can be most relevant if the $T_{1}$ is not known a priori. Continuous flow between the column and NMR probe could be an alternative, ${ }^{22,23}$ although the proposed setup has the advantage that the chromatography and NMR methodological parameters, including flow rates, solvents and volumes, remain independent and it allows each step to be optimised separately.

In summary this work is the first demonstration, to our knowledge, of NMR combined with the "modern" generation of chromatography instruments. A SFC protocol for controlled and efficient fractionation is combined with a stripline NMR probe whose absolute sensitivity exceeds that of a commercial $5 \mathrm{~mm}$ NMR cryoprobe system operating at the same magnetic field strength, while at a fraction of the cost. The present results show rapid separation and molecular structural assignment of sub-microgram mass quantities. A future automated setup may substantially improve the quantitative and reproducibility aspects. We anticipate the low cost and high operational flexibility to be convenient in many situations where external calibration standards are unavailable: petrochemicals, polymers, biofluids, metabolomics and natural product analyses. It may also be extended to double-chromatographic separation-extractions in supercritical media, e.g. SFC $\times \mathrm{SFC}$, and SFE-SFC.

\section{Acknowledgements}

This work was supported by the UltraSense NMR project jointly funded by the European Union (EFRO), Gelderland and Overijssel (GO) and Radboud University Nijmegen (RU). We are grateful to Gerrit Janssen, Hans Janssen and Jan van Os (RU) for technical help. We also thank Isabelle Francois (Waters BeNeLux, Belgium) for support and discussions.

\section{References}

1 J. R. Kesting, K. T. Johansen and J. W. Jaroszewski, $A d v$. Biomed. Spectrosc., 2011, 3, 413-434. 
2 L. A. Cardoza, V. K. Almeida, A. Carr, C. K. Larive and D. W. Graham, Trends Anal. Chem., 2003, 22, 766-775.

3 K. Albert, NMR as a chromatography detector, in Handbook of Spectroscopy, Enlarged edn, Wiley-VCH, Weinheim, Germany, 2nd edn, 2014.

4 D. L. Olson, T. L. Peck, A. G. Webb, R. L. Magin and J. V. Sweedler, Science, 1995, 270, 1967-1969.

5 A. G. Webb, Prog. NMR Spectrosc., 1997, 31, 1-42.

6 R. Subramanian, M. M. Lam and A. G. Webb, J. Magn. Reson., 1998, 133, 227-231.

7 R. M. Fratila, M. V. Gomez, S. Sykora and A. H. Velders, Nat. Commun., 2014, 5, 3025.

8 P. J. M. van Bentum, J. W. G. Janssen, A. P. M. Kentgens, J. Bart and J. G. E. Gardeniers, J. Magn. Reson., 2007, 189, 104-113.

9 A. P. M. Kentgens, J. Bart, P. J. M. van Bentum, A. Brinkmann, E. R. H. van Eck, J. G. E. Gardeniers, J. W. G. Janssen, P. Knijn, S. Vasa and M. H. W. Verkuijlen, J. Chem. Phys., 2008, 128, 052202.

10 J. Bart, J. W. G. Janssen, P. J. M. van Bentum, A. P. M. Kentgens and J. G. E. Gardeniers, J. Magn. Reson., 2009, 201, 175-185.

11 J. Bart, A. J. Kolkman, A. J. Oosthoek de Vries, K. Koch, P. J. Nieuwland, J. W. G. Janssen, P. J. M. van Bentum, K. A. M. Ampt, F. P. T. J. Rutjes, S. S. Wijmenga,
J. G. E. Gardeniers and A. P. M. Kentgens, J. Am. Chem. Soc., 2009, 131, 5014-5015.

12 D. Falck, A. J. Oosthoek de Vries, A. J. Kolkman, H. Lingeman, M. Honing, S. S. Wijmenga, A. P. M. Kentgens and W. M. A. Niessen, Anal. Bioanal. Chem., 2013, 405, 6711-6720.

13 D. R. Gere, Science, 1983, 222, 253-259.

14 K. Albert, J. Chromatogr. A, 1997, 785, 65-83.

15 C. R. Yonker and J. C. Linehan, Prog. Nucl. Magn. Reson. Spectrosc., 2005, 47, 95-109.

16 C. J. Hudalla and P. D. McDonald, Chromatogr. Today, 2012, 5, 18-20.

17 Waters Acquity ${ }^{\mathrm{TM}} \mathrm{UPC}^{2}$. Instrument documentation available at http://www.waters.com/upc2.

18 M. Krucker, A. Lienau, K. Putzbach, M. D. Grynbaum, P. Schuler and K. Albert, Anal. Chem., 2004, 76, 2623-2628.

19 Arduino, an open-source microcontroller platform. See http://www.arduino.cc/.

20 J. K. Baker and C. W. Myers, Pharm. Res., 1991, 8, 763-770.

21 M. Frenkel and K. N. Marsh, Spectral data for steroids, TRC data series.

22 On-line LC-NMR and related techniques, ed. K. Albert, John Wiley \& Sons Ltd., Chichester, United Kingdom, 2002.

23 R. J. Lewis, M. A. Bernstein, S. J. Duncan and C. J. Sleigh, Magn. Reson. Chem., 2005, 43, 783-789. 